BTS GUIDELINES

\title{
BTS recommendations for assessing risk and for managing Mycobacterium tuberculosis infection and disease in patients due to start anti-TNF- $\alpha$ treatment \\ British Thoracic Society Standards of Care Committee*
}

Thorax 2005;60:800-805. doi: 10.1136/thx.2005.046797

Guidelines have been compiled by The Joint Tuberculosis Committee of the British Thoracic Society to quantify the risks of reactivation of tuberculosis with anti-tumour necrosis factor $\alpha$ (anti-TNF- $\alpha$ ) treatment. These guidelines are intended to inform respiratory physicians, gastroenterologists, rheumatologists and dermatologists, together with specialist nurses in those disciplines.

See end of article for authors' affiliations

Correspondence to: Professor L P Ormerod, Professor of Respiratory Medicine, Blackburn Royal Infirmary, Blackburn, Lancashire BB2 3LR, UK; pandpormerod@hotmail. com

Received 15 May 2005 Accepted 26 June 2005 Published Online First 29 July 2005

$\mathrm{n}$ view of the increased risk of reactivation of tuberculosis (TB) with anti-tumour necrosis factor $\alpha$ (anti-TNF- $\alpha$ ) treatment, various professional groups have sought the advice of the Joint Tuberculosis Committee of the British Thoracic Society. The objectives of these guidelines are to quantify these risks and to give advice on the treatment of ТВ disease and infection in patients being assessed for antiTNF- $\alpha$ treatment. The risks and benefits of treatment for latent TB infection in those unsuitable for tuberculin skin testing are also covered. These guidelines are intended to inform respiratory physicians, gastroenterologists, rheumatologists and dermatologists, together with specialist nurses in those disciplines. system: grades of recommendation

A At least one meta-analysis, systematic review, or RCT rated as $1^{++}$and directly applicable to the target population; or

A systematic review of RCTs or a body of evidence consisting principally of studies rated as $1^{+}$directly applicable to the target population and demonstrating overall consistency of results.

B A body of evidence including studies rated as $2^{++}$directly applicable to the target population and demonstrating overall consistency of results; or Extrapolated evidence from studies rated as $1^{++}$or $1^{+}$

C A body of evidence including studies rated as $2^{+}$directly applicable to the target population and demonstrating overall consistency of results; or Extrapolated evidence from studies rated as $2^{+}$

D Evidence level 3 or 4 ; or Extrapolated evidence from studies rated as $2^{+}$.
Table 1 Revised SIGN grading

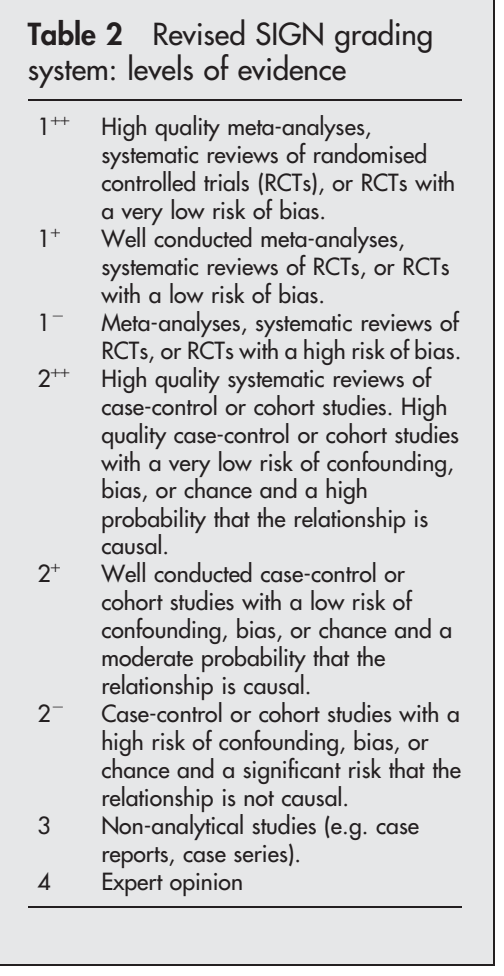

\section{INTRODUCTION}

\subsection{Structure of the recommendations}

The format follows that used for other BTS guidelines. At the start there is a summary of the abstracted bullet points from each section. Following that there is an algorithm summarising the management of patients due to start antiTNF- $\alpha$ treatment (fig 1 ). The recommendations use the revised Scottish Intercollegiate Guidelines Network (SIGN) grading system available at http://www.sign.ac.uk/guidelines/ fulltext/50/section6.html (table 1). The primary source literature has been individually graded for its methodology (where appropriate) and the grading is given alongside the reference using the revised SIGN levels of evidence (table 2).

\subsection{Methodology for the generation of the} recommendations

The initial systematic literature search (Pubmed, EMBASE) was carried out by one of the

* Members of the BTS Standards of Care Subcommittee: L P Ormerod (Chair), H J Milburn, S Gillespie, J Ledingham, D Rampton. 


\section{Abstracted bullet points}

- In patients receiving anti-TNF- $\alpha$ treatment there is an increased risk of clinical tuberculosis (TB) developing.

- Patients should have a clinical examination, their history of any prior TB treatment checked, a chest radiograph and, if appropriate, a tuberculin test (see text). Any patient with an abnormal chest radiograph or previous history of TB or TB treatment should be referred for assessment by a specialist with an interest in TB. (D)

- Patients with an abnormal chest radiograph and/or symptoms raising a suspicion of TB should be thoroughly investigated to exclude active disease. (D)

- Any patient with active TB, either pulmonary or non-pulmonary, should receive standard chemotherapy. (A)

- Patients with active TB should receive a minimum of 2 months full chemotherapy directed by a specialist in TB before starting anti-TNF- $\alpha$ treatment. (D)

- Patients with an abnormal chest radiograph consistent with past TB, or a history of prior extrapulmonary TB but who have received previous adequate treatment, should be monitored regularly. (D)

- Active TB should be excluded by appropriate investigations in patients with an abnormal chest radiograph, or a history of prior pulmonary or extrapulmonary TB not previously adequately treated. Chemoprophylaxis should be given before commencing anti-TNF- $\alpha$ treatment. (A)

- For patients with a normal chest radiograph who are not on immunosuppressive therapy, a tuberculin test is helpful in management: an algorithm is supplied. (D)

- For those with a normal chest radiograph who are on immunosuppressive therapy, a tuberculin test will not be helpful and need not be undertaken. An individual risk assessment should be made (tables 3-5); if the annual risk of TB is greater than the risk of drug induced hepatitis, then chemoprophylaxis should be given. (C) If the risk of hepatitis is greater, the patient should be monitored regularly with suggestive symptoms promptly investigated to permit early diagnosis of active disease. An algorithm is supplied. (C) In general, black African patients aged over 15 years and all South Asians born outside the UK should be considered for chemoprophylaxis with isoniazid for 6 months $(6 \mathrm{H})$.

- Close cooperation between clinicians prescribing anti-TNF- $\alpha$ and specialists in the management of TB is strongly recommended. (D)

committee (LPO) using tuberculosis and biologics 1997-2003 and chemoprophylaxis for TB and hepatitis 1966-2002 as search criteria. A paper based exploration of the relevant literature was pursued from this core dataset. Only English language literature including clinical trials and all well formulated clinical case series were included. Isolated case reports and abstracts were excluded.

After the data appraisal the guideline was initially drafted by LPO, and then discussed by the whole group, the evidence debated, and redrafted several times. The draft was based where possible on the published evidence, but this was combined with clinical expertise where required. The resulting draft was a blend of published evidence and clinical expertise. The manuscript was then placed on the BTS website for consultation by the membership; it was also reviewed by the British Societies for Rheumatology and Gastroenterology. Following this, further amendments took place and the document was reviewed by the Joint Tuberculosis and Standards of Care Committees of the BTS. After final approval from this Committee, the guidelines were submitted for peer review prior to publication

\subsection{Conflict of interest}

All members of the Guideline Committee were asked to submit a written record of possible conflicts of interest to the Standards of Care Committee of the BTS. None were recorded.

\subsection{Suggested review date}

The Guidelines Committee suggest that the guidelines should be reviewed in summer 2008 .

\section{BACKGROUND}

\subsection{The need for recommendations}

The increase in active TB associated with anti-TNF treatment has led to a requirement to screen for active and latent TB in patients before anti-TNF- $\alpha$ treatment is given. This screening, suggested by manufacturers to include tuberculin skin testing, is further complicated by the fact that many patients - up to $79 \%$ in the infliximab study ${ }^{1}$-were receiving immunosuppressive therapy such as maintenance prednisolone, azathioprine, 6-mercaptopurine, methotrexate, cyclophosphamide, ciclosporine, tacrolimus, or mycophenolate mofetil which would interfere with the accuracy of tuberculin skin testing. ${ }^{2}{ }^{3}$ The product licence for infliximab states that the product should be used in conjunction with methotrexate or other immunosuppressants, and in practice the other anti-TNF agents would also normally be used with other immunosuppressants. Chemoprophylaxis or preventive treatment for TB itself carries a small risk, with drug induced hepatitis being the main issue, increasing with age and occasionally fatal. It is also important to exclude active TB disease before chemoprophylaxis is given, particularly as single agent chemoprophylaxis given when active disease is present could lead to the development of drug resistance. These problems have led to many requests for advice in this area.

\subsection{Background epidemiology}

Infliximab, a murine human chimeral monoclonal antibody against TNF- $\alpha$, is approved in the USA and Europe for the treatment of rheumatoid arthritis ${ }^{4}$ and Crohn's disease ${ }^{5}$ and ankylosing spondylitis. Etanercept is a fusion protein that binds free TNF- $\alpha$ using the soluble portion of tumour necrosis factor receptor 2 (TNFR2) coupled with an Fc moiety, ${ }^{6}$ and is also used in the treatment of rheumatoid arthritis, ankylosing spondylitis, psoriatic arthropathy, and juvenile idiopathic arthritis. Adalimumab, a recombinant fully humanised monoclonal antibody against TNF, became licensed for the treatment of rheumatoid arthritis in September $2003 .{ }^{7}$

Post-marketing surveillance in the USA and beyond to the end of May 2001 showed 70 cases of TB associated with infliximab use and a much lower number of cases with etanercept. ${ }^{1}$ Cases of TB have also been described with 


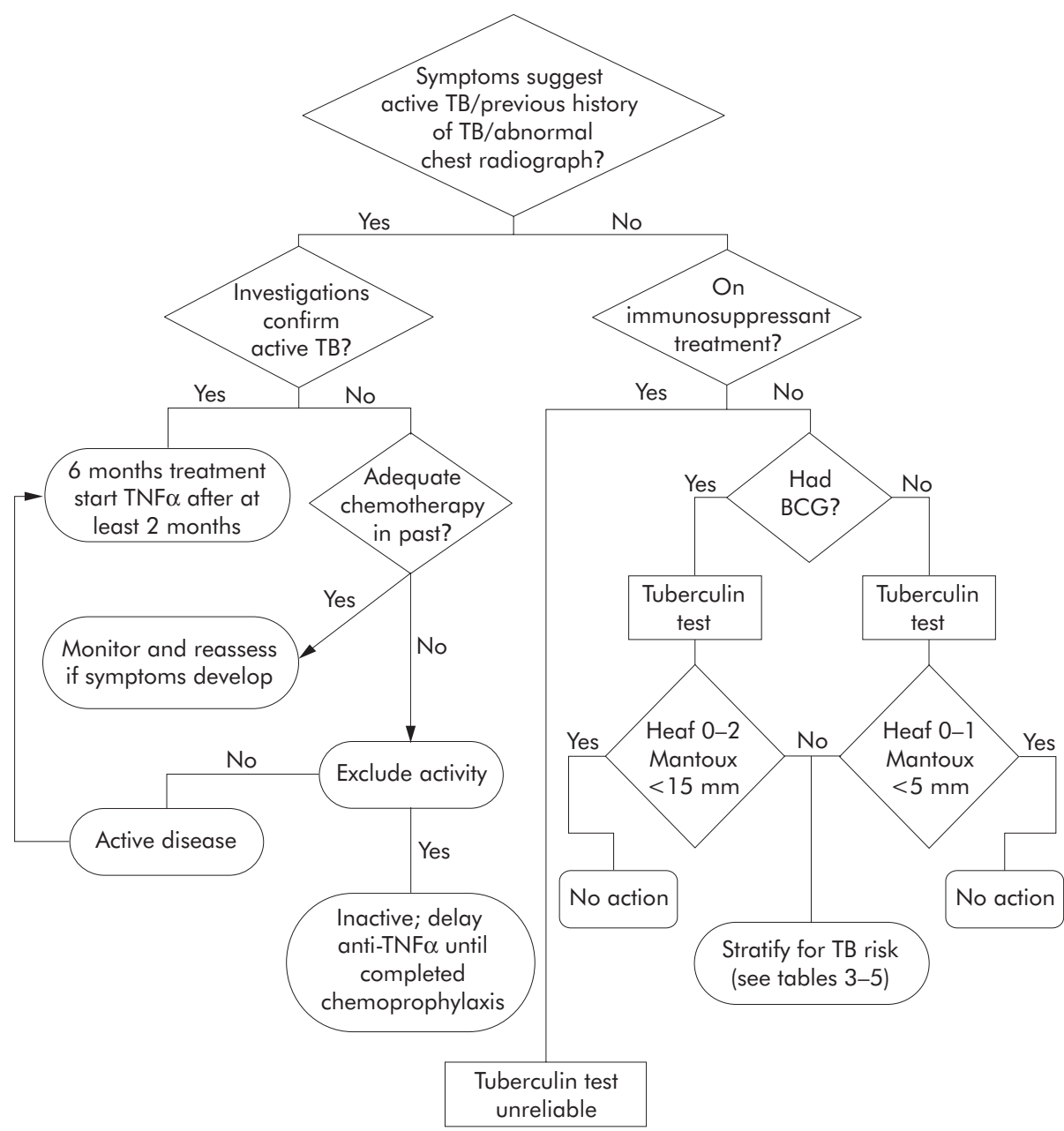

Figure 1 Algorithm for management of TB in patients scheduled for anti-TNF- $\alpha$ treatment.

Notes for algorithm

Mantoux equivalence for Heaf tests: $0-5 \mathrm{~mm}$ induration = Heaf grade $0-1 ; 6-14 \mathrm{~mm}$ induration $=$ Heaf grade $2: \geqslant 15 \mathrm{~mm}$ induration $=$ Heaf grade 3-4.

Where the tuberculin test is unreliable, this should not be performed and patients should be stratified for TB risk (see tables 3-5).

Give chemoprophylaxis if TB risk (tables 3-5) greater than chemoprophylaxis (table 6). In general, black Africans aged over 15 and all South Asians born outside the UK should be considered for chemoprophylaxis with isoniazid for 6 months $(6 \mathrm{H})$.

If the risk from chemoprophylaxis is greater than the TB risk, repeat the chest radiograph within 3 months of starting anti-TNF treatment/investigate if required.

All patients on TB treatment or prophylaxis should be managed by a thoracic or infectious disease physician. Clinical awareness of the possibility of TB should be maintained throughout anti-TNF treatment and for a period up to 6 months after cessation of anti-TNF treatment.

adalimumab. ${ }^{8}$ The majority of the cases of TB in patients given infliximab occurred within three cycles of treatment, with a median of 12 weeks after commencing treatment. ${ }^{7}$ The frequency of tuberculosis was much higher than other opportunist infections reported with the drug, and was higher than reported background rates. ${ }^{9}$ Over $50 \%$ of reported TB cases associated with anti-TNF- $\alpha$ treatment are extrapulmonary. ${ }^{9}$ The number of cases of TB reported to the US Food and Drugs Administration had risen to 117 by December 2001, giving a recalculated TB prevalence of 4l/ 100000 in infliximab/etanercept treated patients in the USA with rheumatoid arthritis, 9/100 000 in patients in the USA with Crohn's disease, and 224/100 000 in all non-USA patients with rheumatoid arthritis or Crohn's disease. ${ }^{10}$ The number of TB cases now reported has reached 242 (Keane J, personal communication, 2004), but the curve has levelled off due to either improved TB risk assessment, tuberculin testing and isoniazid prophylaxis, and/or reporting fatigue.

The estimated prevalence of $\mathrm{TB}$ in rheumatoid arthritis patients in the USA is $6.2 / 100000$ (95\% CI 1.6 to 34$){ }_{i}^{11}$ in patients receiving infliximab the prevalence was originally
24.4/100 000, ${ }^{1}$ approximately a fourfold increase. In a second part of the prevalence study ${ }^{11}$ the incidence of $\mathrm{TB}$ in infliximab treated patients was 52.5/100 000 (95\% CI 14.3 to 134.4), an approximate eightfold increase, but this was based on only a very small number of TB cases. Overall, the subcommittee believes that there is an average fivefold increased risk of TB with anti-TNF- $\alpha$ treatment. (D)

\section{RISKS OF TUBERCULOSIS AND OF DRUG INDUCED HEPATITIS FROM CHEMOPROPHYLAXIS}

\subsection{Risks of TB}

The incidence of TB varies markedly within the United Kingdom (UK) according to a number of factors. ${ }^{12}{ }^{13}$ These are age, ethnic group and, for those not born in the UK, the length of time since first entry. Where possible, data on the current annual risk of TB have been updated from those derived from continuous enhanced surveillance (tables 3-5).

Calculation of the risk of TB is as follows (see also table 5):

- If white and UK born, use data from table 3.

- If Indian subcontinent (ISC), use table 4. 
Table 3 Annual risk of TB disease/100 000 in England and Wales: effect of age (to the nearest whole number)

\begin{tabular}{lcl}
\hline Age (years) & White & Black African \\
\hline $0-14$ & 1 & 47 \\
$15-34$ & 2 & 314 \\
$35-54$ & 4 & 168 \\
$55-74$ & 7 & 204 \\
$>75$ & 11 & Not available \\
\hline
\end{tabular}

Population figures from the Office of National Statistics Labour Force Survey 2000.

TB data from case reports to Enhanced TB Surveillance 2000 Health Protection Agency.

- If black African, use table 3 (similar data to table 4 not yet available).

- If either white, non-UK born, or other ethnic group, use "All patient rate" in table 4.

- If in doubt or in special circumstances, consult local thoracic physician.

\subsection{Risks of drug induced hepatitis from TB chemoprophylaxis}

A database search (Medline and Embase) was carried out on the reported hepatotoxicity of antituberculosis chemoprophylaxis from 1966 to 2002 in adults. Children were excluded because they have a very low rate of drug reactions, and studies in HIV positive individuals were excluded because such individuals have a higher than normal drug reaction profile. ${ }^{14}$ The hepatitis rates for various regimens were derived from these data (table 6).

Only hepatitis sufficient to stop treatment (symptomatic) or grade 3 alanine transferase (ALT 5-20 times normal) or grade 4 (ALT $>20$ times normal) hepatitis is reported here. According to the manufacturers, the use of anti-TNF treatment is contraindicated if there is co-infection with HIV, hepatitis B or C, so this guidance does not apply to patients with these co-infections.

\section{RECOMMENDATIONS}

\subsection{Assessment before anti-TNF treatment}

Patients should have a clinical examination, their history of any prior TB treatment checked, a chest radiograph and, if appropriate, a tuberculin test (sections 4.4 and 4.5). Any patient with an abnormal chest radiograph or previous history of $\mathrm{TB}$ or $\mathrm{TB}$ treatment should be referred to a specialist with an interest in $\mathrm{TB}$, either a thoracic or infectious disease physician. Close cooperation between clinicians prescribing anti-TNF treatment and specialists in the management of TB is strongly recommended. (D)
Table 5 Sample calculations based on data in tables 3 and 4

\begin{tabular}{|c|c|c|c|c|}
\hline Case type & $\begin{array}{l}\text { Annual risk } \\
\text { of TB } \\
\text { disease/ } \\
100000\end{array}$ & $\begin{array}{l}\text { TB risk } \\
\text { adjusted } \times 5 \\
\text { for anti-TNF } \\
\text { effect }\end{array}$ & $\begin{array}{l}\text { Risks of } \\
\text { prophylaxis } \\
/ 100000 \\
\text { (table 6) }\end{array}$ & $\begin{array}{l}\text { Risk/benefit } \\
\text { conclusion }\end{array}$ \\
\hline $\begin{array}{l}\text { White } \\
\text { Age 55-74 } \\
\text { UK born }\end{array}$ & 7 & 35 & 278 & Observation \\
\hline $\begin{array}{l}\text { ISC } \\
\text { Age }>35 \\
\text { In UK } 3 \text { years }\end{array}$ & 593 & 2965 & 278 & Prophylaxis \\
\hline $\begin{array}{l}\text { Black African } \\
\text { Age 35-54 }\end{array}$ & 168 & 840 & 278 & Prophylaxis \\
\hline $\begin{array}{l}\text { Other ethnic } \\
\text { Age } 35+ \\
\text { In UK > } 5 \text { years }\end{array}$ & 39 & 195 & 278 & Observation \\
\hline \multicolumn{5}{|c|}{$\begin{array}{l}\text { The weighted average risk for prophylaxis with isoniazid }(6 \mathrm{H}) \text { is } 278 / \\
100000 \text { which is used for these calculations. The weighted average risk } \\
\text { for rifampicin/isoniazid (3RH is higher at } 1766 / 100000 \text {, but this } \\
\text { regimen may need to be considered if a shorter duration of } \\
\text { chemoprophylaxis is needed on clinical grounds (see section 4.6). }\end{array}$} \\
\hline
\end{tabular}

\subsection{Active TB found before anti-TNF- $\alpha$ treatment}

Patients with chest radiographic abnormalities (such as infiltrates, cavitation, pleural effusion or mediastinal lymphadenopathy) and/or symptoms raising a suspicion of TB should be thoroughly investigated to exclude active disease. This should include sputum microscopy and culture for acidfast bacilli and, if indicated, bronchoscopy and washings and biopsy and culture of pleura and/or mediastinum. Extrapulmonary sites may require material to be obtained by aspiration for culture or biopsy for culture and histological examination. (D)

Any person with active TB, either pulmonary or nonpulmonary, should receive standard chemotherapy. ${ }^{15}$ (A) Anti-TNF- $\alpha$ treatment should not be commenced for at least 2 months after antituberculosis treatment with full compliance has begun, supervised by a thoracic physician or infectious disease specialist, and until the drug susceptibility profile of the organism in those with positive cultures is known, as a minimum. (D) Ideally, it would be preferable to delay anti-TNF treatment until completion of a full course of antituberculosis treatment. (D)

\subsection{Inactive TB found before anti-TNF- $\alpha$ treatment}

(a) Previous adequate treatment

Patients with an abnormal chest radiograph consistent with past TB or a history of prior extrapulmonary TB who have received previous adequate treatment, as judged by a thoracic or infectious disease physician, can start anti-TNF- $\alpha$ treatment but should be monitored clinically every 3 months with

Table 4 Annual risk of TB disease/100 000 in England and Wales: effect of place of birth/duration of residence in England and Wales

\begin{tabular}{lllrc}
\hline Age & Place of birth & Years after first entry & All patient rate & ISC ethnic rate \\
\hline $0-14$ & UK & & 3 & 21 \\
& Abroad & & 31 & 88 \\
$15+$ & UK & $0-4$ & 4 & 59 \\
$15-34$ & Abroad & $5+$ & 180 & 540 \\
& & $0-4$ & 146 & 87 \\
$35+$ & Abroad & $5+$ & 39 & 593 \\
& & & & 108 \\
\hline
\end{tabular}

ISC, Indian subcontinent.

Population figures from the Office of National Statistics Labour Force Survey 2000.

TB data from case reports to Enhanced TB Surveillance 2000 Health Protection Agency. 
Table 6 Hepatic risks of chemoprophylaxis

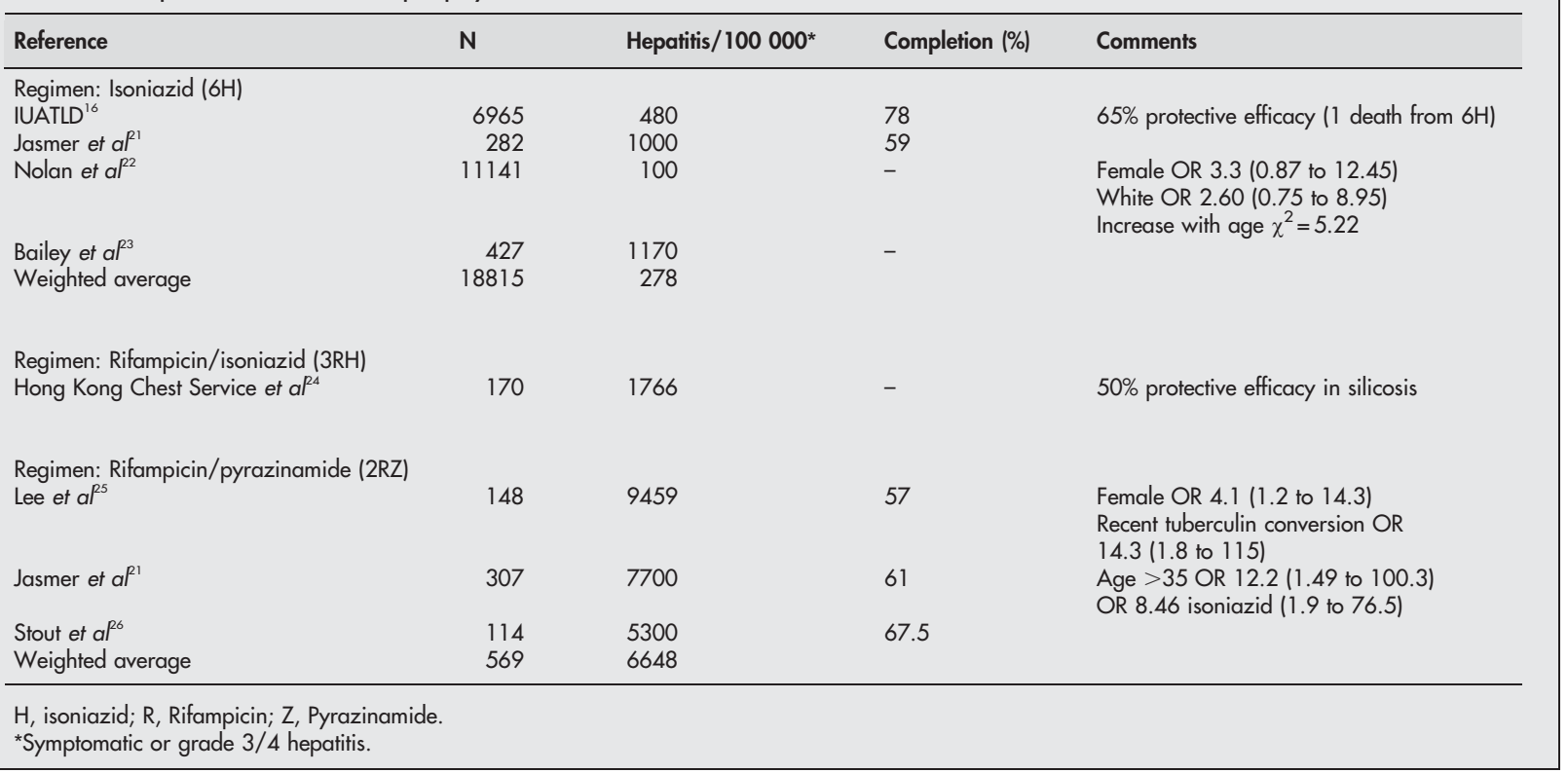

a chest radiograph and sputum cultures if respiratory symptoms develop. (D)

The onset of new respiratory symptoms, particularly within 3 months of commencing anti-TNF treatment, should be investigated promptly. (D)

\section{(b) Previous inadequate treatment}

Patients with an abnormal chest radiograph or a history of prior pulmonary or extrapulmonary TB not previously adequately treated should have active TB excluded by appropriate investigations (section 4.2) under the care of a thoracic or infectious disease physician. In such individuals, even when active disease has been excluded, the annual risk of TB (reactivation) is much higher than in the general population rate, ${ }^{16}$ so the risk-benefit analysis strongly favours chemoprophylaxis (section 4.6). (A)

Ideally, chemoprophylaxis for $\mathrm{TB}$ for patients in this category should be completed before starting anti-TNF treatment. If the clinician is concerned about the potential 6 month delay in starting such treatment, discussion should be held with the patient and the clinician supervising chemoprophylaxis about using a shorter (but potentially more toxic) chemoprophylaxis regimen (section 4.6). (D)

\subsection{Patients with a normal chest radiograph assessable by skin tuberculin test}

For patients with a normal chest radiograph who are not on immunosuppressive therapy, a tuberculin test is helpful in management. The tuberculin test needs to be interpreted in the light of the BCG history. In those without a BCG history, Heaf grades 0-1 (Mantoux 1:10 000 0-5 mm) are negative but Heaf grades $2-4$ (Mantoux 1:10 $000 \geqslant 6 \mathrm{~mm}$ ) are positive and should lead to a risk assessment. In those with a prior BCG history confirmed by scar or adequate documentation, Heaf 0-2 grades (Mantoux 1:10 $000 \quad 0-14 \mathrm{~mm}$ ) need no action, but Heaf grades 3-4 (Mantoux 1:10 $000 \geqslant 15 \mathrm{~mm}$ ) may represent either latent infection or BCG effect so require a risk assessment (see algorithm in fig 1). (C)

\subsection{Patients with a normal chest radiograph not assessable by skin tuberculin test}

The majority of potential recipients of anti-TNF- $\alpha$ medication will have a normal chest radiograph and will have been on immunosuppressive therapy, thus hindering the interpretation of tuberculin testing. ${ }^{3}$ In these individuals an individual risk-benefit calculation will be needed. The estimated annual risk of tuberculosis disease (tables 3 and 4) should be multiplied by a factor of 5 to give an annual risk on anti-TNF treatment, ${ }^{1}{ }^{11}$ based on the current reported rates of TB in association with such treatments. (D)

If the calculated annual risk on anti-TNF treatment is higher than the risk of hepatitis, then the risk-benefit analysis favours chemoprophylaxis; if lower, the risk-benefit calculation favours observation and investigation of symptoms. Table 5 gives some worked examples. (C) In general, black Africans aged over 15 years and all South Asians born outside the UK should be considered for chemoprophylaxis with isoniazid for 6 months $(6 \mathrm{H})$.

\subsection{Chemoprophylaxis}

There are two potential chemoprophylaxis regimens: isoniazid for 6 months $(6 \mathrm{H})$ or rifampicin plus isoniazid for 3 months (3RH). Rifampicin and pyrazinamide for 2 months (2RZ) was a regimen used in the USA, ${ }^{17}$ but it had a very high rate of hepatitis (table 6) with a number of fatalities reported. ${ }^{18}{ }^{19}$ Accordingly, the choice of regimen is between $6 \mathrm{H}$ which has a lower hepatitis rate and $3 \mathrm{RH}$ which may have advantages in terms of shorter duration and thus possibly better adherence (table 6), and also less risk of drug resistance developing if active disease is present. ${ }^{20}$ (A)

The decision on the chemoprophylaxis regimen should be made by the thoracic or infectious disease physician following informed discussion with both the patient and the referring clinician. (D)

In contrast to patients falling into category $4.3 \mathrm{~b}$ (above), chemoprophylaxis for patients with a normal chest radiograph not assessable by tuberculin skin tests can start concurrently with anti-TNF treatment. If the chemoprophylaxis regimen contains rifampicin, any maintainance dose of prednisolone should be doubled for its duration and note made of interactions with other immunosuppressive agents such as tacrolimus and cyclosporine. (D)

It should be noted that no chemoprophylaxis regimen is wholly effective; protective efficacies of $60 \%$ have been reported for $6 \mathrm{H}^{27}$ and of $50 \%$ for $3 \mathrm{RH} .{ }^{24}$ If patients who have had chemoprophylaxis develop symptoms suggestive of clinical TB, they should be promptly and appropriately investigated. (D) 


\subsection{Management of clinical TB developing during anti- TNF- $\alpha$ treatment}

Patients who develop active TB while on anti-TNF- $\alpha$ treatment should receive full antituberculosis chemotherapy. ${ }^{15}$ (A) In these circumstances, which are different from TB disease diagnosed before anti-TNF- $\alpha$ treatment has started, the anti-TNF- $\alpha$ treatment can be continued if clinically indicated because the patient would otherwise be prevented from receiving the continued clinical benefit to their underlying disease and may have a flare up or major clinical deterioration. (D) Although there are no good data in this area, it is known that HIV positive individuals with reduced CD4 counts and clinical TB, who are even more immunosuppressed than those on anti-TNF- $\alpha$ treatment, respond just as well to TB treatment as those who are HIV negative. ${ }^{14}$

\section{SUGGESTED AUDIT CRITERIA}

Suggested audit criteria are as follows.

- History of previous TB checked? (Y/N)

- Chest radiograph within 3 months of starting anti-TNF- $\alpha$ treatment? $(\mathrm{Y} / \mathrm{N})$

- Chest radiograph normal? $(\mathrm{Y} / \mathrm{N})$

- If abnormal, referred to TB specialist? (Y/N)

- Tuberculin skin test possible before treatment? $(\mathrm{Y} / \mathrm{N})$

- If no, referred for risk stratification? $(\mathrm{Y} / \mathrm{N})$

- If skin test performed, is it positive? $(\mathrm{Y} / \mathrm{N})$

- If risk stratification performed, given treatment for latent $\mathrm{TB}$ infection? $(\mathrm{Y} / \mathrm{N})$

\section{Authors' affiliations}

L P Ormerod, Consultant Chest Physician, Blackburn Royal Infirmary, Blackburn, Lancs BB2 3LR, UK

H J Milburn, Consultant Chest Physician, Guy's and St Thomas' Hospitals, London SE1 9RT

S Gillespie, Consultant Microbiologist, Department of Medical Microbiology, Royal Free and University College Medical School, London NW3 2PF

J Ledingham, Consultant Rheumatologist, Queen Alexandra Hospital, Portsmouth PO6 3LY (representing the British Society of Rheumatology) D Rampton, Consultant Gastroenterologist, Barts and the London NHS Trust, London E1 1BB (representing the British Society of Gastroenterology)

\section{REFERENCES}

1 Keane J, Gershon S, Wise RP, et al. Tuberculosis associated with infliximab, a tumor necrosis factor-alpha neutralizing agent. N Engl J Med 2001;345:1098-104, (II).

2 Joint Committee on Vaccination and Immunisation. Tuberculin testing. In Vaccination and immunisation. London: HMSO, 1996: Chapter 32

3 Mow WS, Abreu-Martin MT, Papadakis KA, et al. High incidence of anergy in inflammatory bowel disease patients limits the usefulness of PPD screening before infliximab therapy. Clin Gastroenterol Hepatol 2004;2:309-13.
4 Schuna AA, Megeff $C$. New drugs for the treatment of rheumatoid arthritis. Am J Health Syst Pharm 2000;57:225-34.

5 Kornbluth A. Infliximab approved for use in Crohn's disease: a report on the FDAGI Advisory Committee Conference. Inflamm Bowel Dis 1998;4:328-9.

6 Choy EHS, Punayi GS. Cytokine pathways and joint inflammation in rheumatoid arthritis. N Engl J Med 2001;344:907-16.

7 Weinblatt ME, Keystone EC, Furst DE, et al. Adalimumab, a fully human antitumour necrosis factor alpha monoclonal antibody, for the treatment of rheumatoid arthritis in patients taking concomitant methotrexate: the ARMADA trial. Arthritis Rheum 2003;48:855.

8 Anon. HUMIRA prescribing information. North Chicago, IL: Abbott Laboratories, 2002

9 Gardam MA, Keystone EC, Menzies R, et al. Anti-tumour necrosis factor agents and tuberculosis risk: mechanisms of action and clinical management. Lancet Infect Dis 2003;3:148-55.

10 Keane J, Gershon SK, Braun MM. Tuberculosis and treatment with infliximab. N Engl J Med 2002;346:625-6. (II).

11 Wolfe F, Michaud K, Anderson J, et al. Tuberculosis infection with rheumatoid arthritis and the effect of infliximab therapy. Arthritis Rheum 2004;50:372-9. (II).

12 Kumar D, Watson JM, Charlett A, et al. Tuberculosis in England and Wales in 1993: results of a national survey. Thorax 1997;52:1060-7. (II).

13 Rose AMC, Watson JM, Graham C, et al. Tuberculosis at the end of the 20th century in England and Wales: results of a national survey in 1998. Thorax 2001;56:173-9. (II).

14 Pozniak A, Miller R, Ormerod LP. Treatment of tuberculosis in HIV-infected patients. AIDS 1999;13:435-45.

15 Joint Tuberculosis Committee of the British Thoracic Society. Chemotherapy and management of tuberculosis: recommendations 1998. Thorax 1998;53:536-45. (I).

16 International Union against Tuberculosis Committee on Prophylaxis. Efficacy of various durations of isoniazid preventive therapy for tuberculosis: five years of follow-up in the IUAT trial. Bull WHO 1982;60:555-64. (I).

17 American Thoracic Society and Centers for Disease Control and Prevention. Targeted tuberculin testing and treatment of latent tuberculosis infection. Am J Respir Crit Care Med 2000;161:S221-47. (II).

18 Centers for Disease Control. Fatal and severe hepatitis associated with rifampin and pyrazinamide for the treatment of latent tuberculosis infectionNew York and Georgia, 2000. MMWR Morbidity Mortality Weekly Report 2001;50:289-91. (III).

19 Centers for Disease Control. Update: Fatal and severe liver injuries associated with rifampin and pyrazinamide for latent tuberculosis infection, and revisions in the American Thoracic Society/CDC recommendations-United States 2001. MMWR Morbidity Mortality Weekly Report 2001;50:733-5. (II).

20 Joint Tuberculosis Committee of the British Thoracic Society. Control and prevention of tuberculosis: recommendations 2000. Thorax 2000;55:887-901. (II).

21 Jasmer RM, Saukkonen JJ, Blumberg HM, et al. Short-course rifampin and pyrazinamide compared with isoniazid for latent tuberculosis infection: a multicenter clinical trial. Ann Intern Med 2002;137:640-7. (I).

22 Nolan CM, Goldberg SV, Buskin SE. Hepatotoxicity associated with isoniazid preventive therapy: a seven year survey from a public health tuberculosis clinic. JAMA 1999;281:1014-8. (II).

23 Bailey WC, Taylor SL, Dascomb HE, et al. Disturbed hepatic function during isoniazid chemoprophylaxis. Am Rev Respir Dis 1973;107:523-9. (II).

24 Hong Kong Chest Service, Tuberculosis Research Centre Madras, and British Medical Research Council. A double-blind placebo-controlled clinical trial of three antituberculosis chemoprophylaxis regimens in patients with silicosis in Hong Kong. Am Rev Respir Dis 1992;145:36-41. (I).

25 Lee AM, Mennone JZ, Jones RC, Paul WS. Risk factors for hepatotoxicity associated with rifampin and pyrazinamide for the treatment of latent tuberculosis infection: experience from three public health tuberculosis clinics. Int J Tuberc Lung Dis 2002;6:995-1000. (II).

26 Stout JE, Engemann JJ, Cheng AC, et al. Safety of two months rifampin and pyrazinamide for treatment of latent tuberculosis. Am J Respir Crit Care Med 2003;167:824-7. (II)

27 Smieja MJ, Marchetti CA, Cook DJ, et al. Isoniazid for preventing tuberculosis in non-HIV infected persons (Cochrane Review). In: The Cochrane Library, Issue 2, 2004. (I). 